\title{
Spontaneous Relaxation of a Charge Qubit under Electrical Measurement
}

\author{
Xin-Qi Li, ${ }^{1,2}$ Ping Cui, ${ }^{2}$ and YiJing Yan $^{2}$ \\ ${ }^{1}$ State Key Laboratory for Superlattices and Microstructures, Institute of Semiconductors, Chinese Academy of Sciences, \\ P.O. Box 912, Beijing 100083, China \\ ${ }^{2}$ Department of Chemistry, Hong Kong University of Science and Technology, Kowloon, Hong Kong
}

(Received 11 August 2004; published 17 February 2005)

\begin{abstract}
In this work we first derive a generalized conditional master equation for quantum measurement by a mesoscopic detector, then study the readout characteristics of qubit measurement where a number of remarkable new features are found. The work would, in particular, highlight the qubit spontaneous relaxation effect induced by the measurement itself rather than an external thermal bath.
\end{abstract}

DOI: 10.1103/PhysRevLett.94.066803

The recent renewed interest of measuring a two-state quantum system (qubit) stems largely from the rapidly developing field of quantum computing. A possible solidstate implementation of such measurement is to measure a charge qubit by a mesoscopic detector which, for instance, can be either a quantum-point contact (QPC) [1-4] or a single-electron transistor [5].

For a realistic setup of such measurement, the nontrivial correlation between the detector and the qubit has been the focus of recent theoretical studies. However, sometimes the treatment of this correlation is incomplete. For instance, in a number of publications on the qubit measurement by a QPC $[1,4,6,7]$, the energy transfer between the detector and the qubit has been ignored, which leads the qubit to an incorrect statistical mixture under low measurement voltage, as shown in our work [8]. Two recent publications considered the energy-exchange induced inelastic effect on the detector power spectrum by using, respectively, the real-time Green function approach [9] and the quantum jump technique [10], where a number of controversial results were attained and are cause for further debate [11].

In this Letter, by generalizing the work of Gurvitz et al. $[1,3,4]$, we present an alternative approach to study the inelastic effect in the qubit measurement by a QPC. Connections with the previous work will be established in a transparent way, and new features will be illustrated in both the output current and the power spectrum. In particular, we shall highlight the qubit spontaneous relaxation effect induced by energy exchange with the measuring device, instead of coupling to an external thermal bath as discussed in Ref. [4].

For the sake of generality, we first formally consider an arbitrary quantum system measured by a QPC, described by

$$
\begin{aligned}
H & =H_{0}+H^{\prime}, \\
H_{0} & =H_{s}+\sum_{k}\left(\epsilon_{k}^{L} c_{k}^{\dagger} c_{k}+\epsilon_{k}^{R} d_{k}^{\dagger} d_{k}\right), \\
H^{\prime} & =\sum_{k, q}\left[T_{q k}\left\{\left|\psi_{s}\right\rangle\left\langle\psi_{s}\right|\right\} d_{q}^{\dagger} c_{k}+\text { H.c. }\right] .
\end{aligned}
$$

PACS numbers: 73.63.Kv, 03.65.Ta, 03.67.Lx, 85.35.Be

In this decomposition, the free part of the total Hamiltonian $H_{0}$ contains the Hamiltonians of the measured system $H_{s}$ and the QPC reservoirs (the last two terms). The interaction Hamiltonian $H^{\prime}$ describes electron tunneling through the QPC, e.g., from state $|k\rangle$ in the left reservoir to state $|q\rangle$ in the right reservoir, with tunneling amplitude $T_{q k}\left\{\left|\psi_{s}\right\rangle\left\langle\psi_{s}\right|\right\}$ that is conditioned on the eigenstate $\left|\psi_{s}\right\rangle$ of the observable.

Regarding the tunneling Hamiltonian $H^{\prime}$ as perturbation, on the basis of the second-order cummulant expansion we can derive a formal equation for the reduced density matrix as [12]

$$
\dot{\rho}(t)=-i \mathcal{L} \rho(t)-\int_{0}^{t} d \tau\left\langle\mathcal{L}^{\prime}(t) \mathcal{G}(t, \tau) \mathcal{L}^{\prime}(\tau) \mathcal{G}^{\dagger}(t, \tau)\right\rangle \rho(t) .
$$

Here the Liouvillian superoperators are defined as $\mathcal{L}(\cdots) \equiv\left[H_{s},(\cdots)\right], \mathcal{L}^{\prime}(\cdots) \equiv\left[H^{\prime},(\cdots)\right]$, and $G(t, \tau) \times$ $(\cdots) \equiv G(t, \tau)(\cdots) G^{\dagger}(t, \tau)$, with $G(t, \tau)$ the usual propagator (Green's function) associated with $H_{s}$. The reduced density matrix $\rho(t)=\operatorname{Tr}_{D}\left[\rho_{T}(t)\right]$, resulting from tracing out all the detector degrees of freedom from the entire density matrix. However, for quantum measurement where the specific readout information is likely to be recorded, the average should be performed over the unique class of states of the detector we are trying to keep track of.

For the measurement setup under study, the relevant quantity of readout is the transport current $i(t)$ in the detector, or equivalently, the number of electrons that have tunnelled through the detector, $n(t)=\int_{0}^{t} d t^{\prime} i\left(t^{\prime}\right)$. We therefore classify the Hilbert space of the detector as follows. First, we define the subspace in the absence of electron tunneling through the detector as $D^{(0)}$, which is spanned by the product of all many-particle states of the two isolated reservoirs, formally denoted as $D^{(0)} \equiv$ $\operatorname{span}\left\{\left|\Psi_{L}\right\rangle \otimes\left|\Psi_{R}\right\rangle\right\}$. Then, we introduce the tunneling operator $f^{\dagger} \sim f_{q k}^{\dagger}=d_{q}^{\dagger} c_{k}$, and denote the Hilbert subspace corresponding to $n$ electrons tunnelled from the left to the right reservoirs as $D^{(n)}=\left(f^{\dagger}\right)^{n} D^{(0)}$, where $n=1,2, \ldots$ The entire Hilbert space of the detector is $D=\oplus_{n} D^{(n)}$.

With the above classification of the detector states, the average over states in $D$ in Eq. (2) is replaced with states in 
the subspace $D^{(n)}$, leading to a conditional master equation

$$
\dot{\rho}^{(n)}(t)=-i \mathcal{L} \rho^{(n)}(t)-\int_{0}^{t} d \tau \operatorname{Tr}_{D^{(n)}}\left[\mathcal{L}^{\prime}(t) \mathcal{G}(t, \tau) \mathcal{L}^{\prime}(\tau) \mathcal{G}^{\dagger}(t, \tau) \rho_{T}(t)\right] .
$$

Here $\rho^{(n)}(t)=\operatorname{Tr}_{D^{(n)}}\left[\rho_{T}(t)\right]$, which is the reduced density matrix of the measured system conditioned by the number of electrons tunnelled through the detector until time $t$. Now we transform the Liouvillian operator product in Eq. (3) into the conventional Hilbert form:

$$
\begin{aligned}
\mathcal{L}^{\prime}(t) G(t, \tau) \mathcal{L}^{\prime}(\tau) \mathcal{G}^{\dagger}(t, \tau) \rho_{T}(t) & =\left[H^{\prime}(t) G(t, \tau) H^{\prime}(\tau) G^{\dagger}(t, \tau) \rho_{T}(t)-G(t, \tau) H^{\prime}(\tau) G^{\dagger}(t, \tau) \rho_{T}(t) H^{\prime}(t)\right]+\text { H.c. } \\
& \equiv[I-I I]+\text { H.c. }
\end{aligned}
$$

For the convenience of description, we rewrite the interaction Hamiltonian as $H^{\prime}(t)=Q F(t)$. Here we have assumed the tunneling amplitude $T_{k q}$ to be real and independent of the reservoir state " $k q$ " and denoted it by $Q$, which depends on the state of the measured system. The detector fluctuation is described by $F(t) \equiv f(t)+f^{\dagger}(t)$, with $f \equiv \sum_{k q} c_{k}^{\dagger} d_{q}$ and $f^{\dagger} \equiv \sum_{k q} d_{q}^{\dagger} c_{k}$. To proceed, two physical considerations are further involved as follows: (i) Instead of the conventional Born approximation for the entire density matrix $\rho_{T}(t) \simeq \rho(t) \otimes \rho_{D}$, we propose the ansatz $\rho_{T}(t) \simeq \sum_{n} \rho^{(n)}(t) \otimes \rho_{D}^{(n)}$, where $\rho_{D}^{(n)}$ is the density operator of the detector reservoirs with $n$ electrons tunnelled through the detector. With the ansatz of the density operator, tracing over the subspace $D^{(n)}$ yields

$$
\operatorname{Tr}_{D^{(n)}}[I]=\operatorname{Tr}_{D}\left[F(t) F(\tau) \rho_{D}^{(n)}\right]\left[Q G(t, \tau) Q G^{\dagger}(t, \tau) \rho^{(n)}\right]
$$

$$
\begin{aligned}
\operatorname{Tr}_{D^{(n)}}[I I]= & \operatorname{Tr}_{D}\left[f^{\dagger}(\tau) \rho_{D}^{(n-1)} f(t)\right]\left[G(t, \tau) Q G^{\dagger}(t, \tau) \rho^{(n-1)} Q\right] \\
& +\operatorname{Tr}_{D}\left[f(\tau) \rho_{D}^{(n+1)} f^{\dagger}(t)\right] \\
& \times\left[G(t, \tau) Q G^{\dagger}(t, \tau) \rho^{(n+1)} Q\right]
\end{aligned}
$$

Here we have utilized the orthogonality between states in different subspaces, which in fact leads to the term selection from the entire density operator $\rho_{T}$. (ii) $\mathrm{Be}-$ cause of the closed nature of the detector circuit, the extra electrons tunnelled into the right reservoir will flow back into the left reservoir via the external circuit. Also, the rapid relaxation processes in the reservoirs will quickly bring the reservoirs to the local thermal equilibrium state determined by the chemical potentials. As a consequence, after the procedure (i.e., the state selection) as done in Eq. (5), the detector density matrices $\rho_{D}^{(n)}$ and $\rho_{D}^{(n \pm 1)}$ in Eq. (5) can be well approximated by $\rho_{D}^{(0)}$, i.e., the local thermal equilibrium reservoir state. Under this consideration, the detector fluctuation correlation functions become, respectively, $\left\langle f^{\dagger}(t) f(\tau)\right\rangle=C^{(+)}(t-\tau), \quad\left\langle f(t) f^{\dagger}(\tau)\right\rangle=$ $C^{(-)}(t-\tau)$, and $\langle F(t) F(\tau)\rangle=C(t-\tau)=C^{(+)}(t-\tau)+$ $C^{(-)}(t-\tau)$. Here, $\langle\cdots\rangle$ stands for $\operatorname{Tr}_{D}\left[(\cdots) \rho_{D}^{(0)}\right]$.

Under the Markovian approximation, the time integral in Eq. (3) is replaced by $\frac{1}{2} \int_{-\infty}^{\infty}$. Substituting Eqs. (4) and (5) into Eq. (3), we obtain

$$
\begin{aligned}
\dot{\rho}^{(n)}= & -i \mathcal{L} \rho^{(n)}-\frac{1}{2}\left\{\left[Q \tilde{Q} \rho^{(n)}+\text { H.c. }\right]\right. \\
& \left.-\left[\tilde{Q}^{(-)} \rho^{(n-1)} Q+\text { H.c. }\right]-\left[\tilde{Q}^{(+)} \rho^{(n+1)} Q+\text { H.c. }\right]\right\} .
\end{aligned}
$$

Here $\tilde{Q}^{( \pm)}=\tilde{C}^{( \pm)}(\mathcal{L}) Q, \quad \tilde{C}^{( \pm)}(\mathcal{L})=\int_{-\infty}^{\infty} d t C^{( \pm)}(t) e^{-i \mathcal{L} t}$, and $\tilde{Q}=\tilde{Q}^{(+)}+\tilde{Q}^{(-)}$. Under the wideband approximation for the detector reservoirs, the spectral function $\tilde{C}^{( \pm)}(\mathcal{L})$ can be explicitly carried out as $[8] \tilde{C}^{( \pm)}(\mathcal{L})=\eta[x /(1-$ $\left.\left.e^{-x / T}\right)\right]_{x=-\mathcal{L} \mp V}$, where $\eta=2 \pi g_{L} g_{R}$ and $T$ is the temperature. In this work we will use the unit system of $\hbar=e=$ $k_{B}=1$. In Eq. (6) the terms in \{\} describe the fluctuation effect of the forward and backward electron tunneling through the detector on the measured system. In particular, the Liouvillian operator " $\mathcal{L}$ " in $\tilde{C}^{( \pm)}(\mathcal{L})$ contains the information of energy transfer between the detector and the measured system, which correlates the energy (spontaneous) relaxation of the measured system with the inelastic electron tunneling in the detector. At the high-voltage limit (i.e., the bias voltage is much larger than the internal characteristic energy difference of the measured system), the spectral function $\tilde{C}^{( \pm)}(\mathcal{L}) \simeq \tilde{C}^{( \pm)}(0)$, and Eq. (6) reduces to the previous result derived in Refs. $[1,3,4,7]$.

In the following, we specify the measured system as a pair of coupled quantum dots (a solid-state charge qubit), described by the Hamiltonian $H_{\mathrm{qu}}=\epsilon_{a}|a\rangle\left\langle a\left|+\epsilon_{b}\right| b\right\rangle \times$ $\langle b|+\Omega(|b\rangle\langle a|+| a\rangle\langle b|)$. Introduce $\epsilon=\left(\epsilon_{a}-\epsilon_{b}\right) / 2$, and set $\left(\epsilon_{a}+\epsilon_{b}\right) / 2$ as the reference energy. The qubit eigenenergies are obtained as $E_{1}=\sqrt{\epsilon^{2}+\Omega^{2}} \equiv \Delta / 2$, and $E_{0}=$ $-\sqrt{\epsilon^{2}+\Omega^{2}}=-\Delta / 2$. Correspondingly, the eigenstates are $|1\rangle=\cos \frac{\theta}{2}|a\rangle+\sin \frac{\theta}{2}|b\rangle$ for the excited state and $|0\rangle=$ $\sin \frac{\theta}{2}|a\rangle-\cos \frac{\theta}{2}|b\rangle$ for the ground state, where $\theta$ is introduced by $\cos \theta=2 \epsilon / \Delta$ and $\sin \theta=2 \Omega / \Delta$. The coupling between the qubit and detector is characterized by $H^{\prime}=$ $Q F$, where $Q=\mathcal{T}+\chi|a\rangle\langle a|$ and $F=\sum_{k, q}\left(c_{k}^{\dagger} d_{q}+\right.$ H.c. $)$.

With the knowledge of $\rho^{(n)}(t)$, one is able to carry out the various readout characteristics of the detector. In the strong projective measurement regime (e.g., $\Omega=0$ ), the measurement-induced wave function collapse of the qubit can be perfectly manifested by the probability distribution function $P(n, t) \equiv \operatorname{Tr}\left[\rho^{(n)}(t)\right]$. Switching on $\Omega$ such that $1 / \Omega$ is comparable to or smaller than the measurement time [5], the qubit state oscillation cannot be read out by a 
series of single shot measurement. In this regime, the continuous weak measurement is an alternative approach to register the qubit oscillations. In the remaining part of this Letter we calculate the output current and noise spectrum based on Eq. (6).

Straightforwardly, the average current flowing through the detector can be generally expressed as

$$
I(t)=\sum_{n} n \operatorname{Tr}\left[\dot{\rho}^{(n)}(t)\right]=\frac{1}{2} \operatorname{Tr}[\bar{Q} \rho Q+\text { H.c. }],
$$

where $\bar{Q} \equiv \tilde{Q}^{(-)}-\tilde{Q}^{(+)}$. For symmetric qubit (i.e., $\epsilon=0$ or $\theta=\pi / 2$ ), the stationary current reads

$$
I_{s}=g_{0} V+g_{1} V\left[1-\frac{\Delta}{V} \frac{G^{(-)}}{G^{(+)}}\right] .
$$

Here $g_{0}=\eta(\mathcal{T}+\chi / 2)^{2}, g_{1}=\eta(\chi / 2)^{2}$, and $G^{( \pm)}=\frac{1}{2} \times$ $\left[F^{(+)}(\Delta, V) \pm F^{(-)}(\Delta, V)\right]$, with $F^{( \pm)}(\Delta, V) \equiv(\Delta \pm V) \times$ $\operatorname{coth}\left(\frac{\Delta \pm V}{2 T}\right)$. We notice that Eq. (8) coincides with the result derived in Ref. [9], but differs from that in Ref. [10]. The former was obtained on the basis of the real-time Green function diagram technique, while the latter resulted from the quantum trajectory technique under rotation-wave approximation. In addition to the measurement current, in the following we detail the studies of output noise spectrum in the regime of continuous weak measurement, where a number of remarkable new features will be revealed.

The noise spectrum can be calculated using MacDonald's formula [4]

$$
S(\omega)=2 \omega \int_{0}^{\infty} d t \sin \omega t \frac{d}{d t}\left[\left\langle n^{2}(t)\right\rangle-(\bar{I} t)^{2}\right],
$$

where $\bar{I}$ is the average current over time and $\left\langle n^{2}(t)\right\rangle=$ $\sum_{n} n^{2} P(n, t)$. It can be shown that

$$
\frac{d}{d t}\left\langle n^{2}(t)\right\rangle=\operatorname{Tr}\left[\bar{Q} \hat{N}(t) Q+\frac{1}{2} \tilde{Q} \rho(t) Q+\text { H.c. }\right],
$$

where $\hat{N}(t) \equiv \sum_{n} n \rho^{(n)}(t)$, which can be calculated via its equation of motion

$$
\frac{d \hat{N}}{d t}=-i \mathcal{L} \hat{N}-\frac{1}{2}\left[Q, \tilde{Q} \hat{N}-\hat{N} \tilde{Q}^{\dagger}\right]+\frac{1}{2}(\bar{Q} \rho Q+\text { H.c. }) \text {. }
$$

For symmetric qubit, it would be desirable to carry out the explicit result. Denoting $S(\omega)=S_{0}+S_{1}(\omega)+S_{2}(\omega)$, the result reads

$$
\begin{aligned}
S_{0} & =2 I_{0} \operatorname{coth} \frac{V}{2 T}+\frac{\chi^{2} \eta}{2}\left[G^{(+)}-\frac{\Delta^{2}}{G^{(+)}}-V \operatorname{coth} \frac{V}{2 T}\right], \\
S_{1}(\omega) & =\left[1-\frac{\Delta}{2 V} \frac{G^{(-)}}{G^{(+)}}\right] \frac{I_{d}^{2} \Gamma_{d} \Delta^{2}}{\left(\omega^{2}-\Delta^{2}\right)^{2}+\Gamma_{d}^{2} \omega^{2}}, \\
S_{2}(\omega) & =\chi^{2} \eta\left[\Gamma_{d} D_{z}+\gamma \bar{I}\right] \frac{G^{(-)}}{\omega^{2}+\Gamma_{d}^{2}} .
\end{aligned}
$$

Here three currents are defined as $I_{0}=\left(I_{a}+I_{b}\right) / 2$, $I_{d}=I_{a}-I_{b}$, and $\bar{I}=I_{0}-\frac{1}{4} \eta \chi^{2} \Delta G^{(-)} / G^{(+)}$, with $I_{a}=$ $\eta(\mathcal{T}+\chi)^{2} V$ and $I_{b}=\eta \mathcal{T}^{2} V$ being the detector currents corresponding to qubit states $|a\rangle$ and $|b\rangle$, respectively. Other quantities in Eq. (12) are introduced as $\Gamma_{d}=\frac{\eta \chi^{2}}{2} G^{(+)}, \gamma=\frac{\eta \chi^{2}}{2} \Delta$, and $D_{z}=-\Delta \sqrt{I_{a} I_{b}} / G^{(+)}-$ $\eta \chi^{2} G^{(-)} / 4$. The three noise spectrum components are, respectively, (i) the zero-frequency noise $S_{0}$, (ii) the Lorentzian spectral function $S_{1}(\omega)$ with a peak around the qubit Rabi frequency $\omega=\Delta$, and (iii) $S_{2}(\omega)$ completely originating from the qubit relaxation induced inelastic tunneling effect in the detector. In addition to $S_{2}(\omega)$, the qubit relaxation also manifests its effect in $S_{0}$ and $S_{1}(\omega)$, i.e., giving rise to the second term of $S_{0}$ and reducing the prefactor in $S_{1}(\omega)$ from unity. If the qubit relaxation induced inelastic effect is neglected, or at the limit of high bias voltage $V \gg \Delta$, Eq. (12) returns to the known result of previous work [6,7].

The measurement-induced relaxation effects of the qubit are further shown in Fig. 1. The major effect of the qubit relaxation shown in Fig. 1(a) is lowering the entire noise spectrum, in qualitative consistence with the finding by Gurvitz et al. [4], where an external thermal bath is introduced to cause qubit relaxation. However, the spontaneous relaxation discussed here does not diminish the telegraph noise peak near zero frequency in the incoherent case, which implies the surviving of the Zeno effect, in contrast to the major conclusion of Ref. [4]. Also, the transition behavior from the coherent to the incoherent regime is different. Figure 1(b) shows the voltage effect on the coherent peak around $\omega=\Delta$. It is found that the peak would be reduced by lowering the measurement voltage. Interestingly, this effect alters the fundamental upper
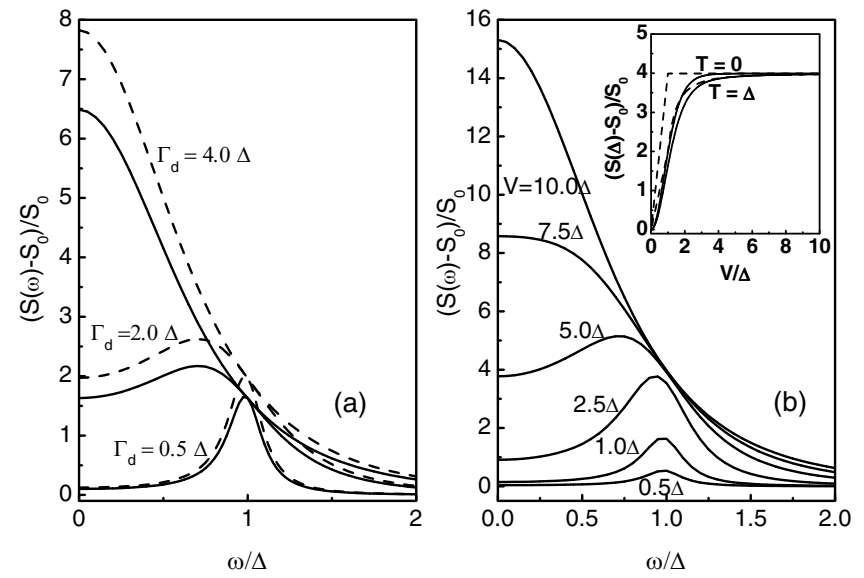

FIG. 1. (a) Noise spectrum in the presence (solid curves) and absence (dashed curves) of the qubit relaxation. (b) Voltage effect on the noise spectrum, particularly on the peak-to-pedestal ratio (inset: where the solid and dashed curves correspond to the presence and absence of the qubit relaxation). The results in (a) and (b) are obtained, respectively, by altering $\chi$ (for a fixed voltage $V=2 \Delta$ ) and the voltage $V$ (for a fixed $\chi=0.1 \Delta$ ). Other parameters are $g_{L}=g_{R}=2.5 / \Delta$ and $T=\Delta$. 
(a) $\theta=\pi / 2$

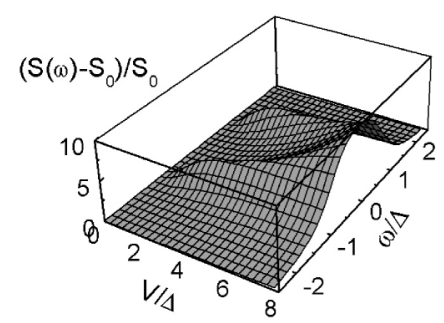

(b) $\theta=\pi / 6$

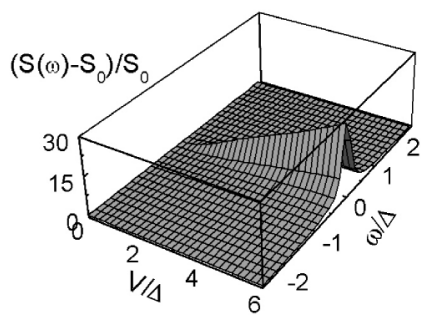

FIG. 2. 3D plot of the noise spectra for (a) the symmetric qubit and (b) the asymmetric qubit. The adopted parameters are $g_{L}=$ $g_{R}=2.5 / \Delta, \chi=0.1 \Delta$, and $T=\Delta$.

bound limit of 4 for the value of the peak-to-pedestal ("signal-to-noise") ratio, $\left[S(\Delta)-S_{0}\right] / S_{0}$, which was found by Korotkov et al. at the high-voltage limit (see the inset) [6].

The voltage effect is further shown in Fig. 2 by the 3D plot of the scaled spectra for different qubit symmetries. In contrast to the present result, we notice that in Ref. [10] no spectral structure was found, i.e., $S(\omega)-S(\infty)=0$, in the wide range of $V<10 \Delta$ for the symmetric qubit $(\theta=$ $\pi / 2$ ). However, Shnirman et al. showed the existence of the coherent peaks at $\omega= \pm \Delta$ for voltage higher than $\Delta$ [9]. For the asymmetric qubit as shown in Fig. 2(b), the coherent peaks at $\omega= \pm \Delta$ are destroyed and a peak around $\omega=0$ is formed. This transition originates from the breakdown of the resonant condition, which replaces the Rabi oscillation of the qubit by incoherent jumping.

Finally, simple analysis in limiting cases can provide additional insight into the correlation between the detector and the qubit. At zero temperature, we obtain a prefactor $1 / 2$ in $S_{1}(\omega)$ in the low-voltage regime $(V<\Delta)$. This result is in sharp contrast to previous conclusions: In Ref. [9] such types of contributions vanish, while in Ref. [10] it does not exist at all in a much wider range of voltage. At the same limit, Eq. (12) also predicts nonvanishing $S_{2}(\omega)$ and nonzero correction to the Schottky shot noise $2 I_{0}$ in $S_{0}$. Remarkably, all these contributions were absent from previous work [9,10], and the reason was attributed to the complete relaxation of the measured qubit to its ground state. Here we understand our distinct result as follows. Consider the key quantity $\left\langle n^{2}(t)\right\rangle=$ $\sum_{n} n^{2} P(n, t)$. Despite the fact that the qubit would relax to its ground state under the concerned limit, the fluctuation of $\left\langle n^{2}(t)\right\rangle$ remains according to Eq. (6), since the conditional qubit state $\rho^{(n)}(t)$ is not at all stationary. In particular, the nonzero off-diagonal elements of $\rho^{(n)}(t)$ contain the information of qubit coherence, which gives rise to the peak structure of the noise spectrum. Therefore, differing from the previous work $[9,10]$ and going beyond the very recent debate [11], we conclude here that at zero temperature and even in low bias voltage $(V \ll \Delta)$, the detector output noise spectrum still has spectral structure, i.e., with excess components in addition to the Schottky shot noise, due to the quantum fluctuations induced by the coupling of the detector and the qubit. This novel feature may deserve further study in future work.

In summary, we have generalized the validity range of the quantum measurement theory developed by Gurvitz et al. to arbitrary voltage and temperatures. The generalized theory properly accounts for the energy transfer between the detector and the measured system. Its application to charge qubit measurement reveals a number of interesting new readout characteristics associated with the new treatment of the correlation between the detector and the qubit.

Support from the National Natural Science Foundation of China, the Major State Basic Research Project No. G001CB3095 of China, and the Research Grants Council of the Hong Kong Government are gratefully acknowledged.

[1] S. A. Gurvitz, Phys. Rev. B 56, 15215 (1997).

[2] I. L. Aleiner, N. S. Wingreen, and Y. Meir, Phys. Rev. Lett. 79, 3740 (1997); Y. Levinson, Europhys. Lett. 39, 299 (1997); L. Stodolsky, Phys. Lett. B 459, 193 (1999); E. Buks, R. Schuster, M. Heiblum, D. Mahalu, and V. Umansky, Nature (London) 391, 871 (1998); S. Pilgram and M. Büttiker, Phys. Rev. Lett. 89, 200401 (2002).

[3] D. Mozyrsky and I. Martin, Phys. Rev. Lett. 89, 018301 (2002).

[4] S. A. Gurvitz, L. Fedichkin, D. Mozyrsky, and G. P. Berman, Phys. Rev. Lett. 91, 066801 (2003).

[5] A. Shnirman and G. Schön, Phys. Rev. B 57, 15400 (1998); Y. Makhlin, G. Schön, and A. Shnirman, Rev. Mod. Phys. 73, 357 (2001); A. A. Clerk, S. M. Girvin, A. K. Nguyen, and A.D. Stone, Phys. Rev. Lett. 89, 176804 (2002).

[6] A. N. Korotkov, Phys. Rev. B 63, 085312 (2001); A. N. Korotkov and D. V. Averin, Phys. Rev. B 64, 165310 (2001); R. Ruskov and A. N. Korotkov, Phys. Rev. B 67, 075303 (2003).

[7] H. S. Goan, G. J. Milburn, H. M. Wiseman, and H. B. Sun, Phys. Rev. B 63, 125326 (2001); H. S. Goan and G. J. Milburn, Phys. Rev. B 64, 235307 (2001).

[8] X. Q. Li, W. K. Zhang, P. Cui, J.S. Shao, Z.S. Ma, and Y. J. Yan, Phys. Rev. B 69, 085315 (2004).

[9] A. Shnirman, D. Mozyrsky, and I. Martin, cond-mat/ 0211618.

[10] T. M. Stace and S. D. Barrett, Phys. Rev. Lett. 92, 136802 (2004); cond-mat/0309610.

[11] D. V. Averin and A. N. Korotkov, Phys. Rev. Lett. 94, 069701 (2005); T. M. Stace and S. D. Barrett, Phys. Rev. Lett. 94, 069702 (2005).

[12] Y. J. Yan, Phys. Rev. A 58, 2721 (1998). 